\title{
THE EFFECTS OF ACTUATION FREQUENCY ON THE SEPARATION CONTROL OVER AN AIRFOIL USING A SYNTHETIC JET
}

\author{
Y. Abe ${ }^{1}$, K. Okada ${ }^{2}$, T. Nonomura ${ }^{3}$, and K. Fujii ${ }^{3}$ \\ ${ }^{1}$ University of Tokyo \\ Sagamihara, Kanagawa 252-5210, Japan \\ ${ }^{2}$ Ryoyu Systems Co. Ltd \\ Nagoya, Aichi 455-0024, Japan \\ ${ }^{3}$ Institute of Space and Astronautical Science, JAXA \\ Sagamihara, Kanagawa 252-5210, Japan
}

\begin{abstract}
The simulation of separation control using a synthetic jet (SJ) is conducted around an NACA (National Advisory Committee for Aeronautics) 0015 airfoil by large-eddy simulation (LES) with a compact difference scheme. The synthetic jet is installed at the leading edge of the airfoil and the effects of an actuation frequency $F^{+}$(normalized by chord length and velocity of freestream) are observed. The lift-drag coefficient is recovered the most for $F^{+}=6$. The relationship between momentum addition by turbulent mixing and large vortex structures is investigated using a phase-averaging procedure based on $F^{+}$. The Reynolds shear stress is decomposed into periodic and turbulent components where the turbulent components are found to be dominant on the airfoil. The strong turbulent components appear near the large vortex structures that are observed in phase- and span-averaged flow fields.
\end{abstract}

\section{INTRODUCTION}

Microdevices for separation control [1,2] have recently drawn significant attention because they consume lesser energy, have simpler structures, and offer greater effective control over unsteady flow fields compared with conventional devices such as "steady jet" and "vortex generator." This paper focuses on a "synthetic jet" device, which is one of the most advanced microdevices for controlling a separated flow. Figure 1 shows a schematic diagram of an SJ. A synthetic jet consists of a cavity with a connected orifice. The bottom of this cavity oscillates with 


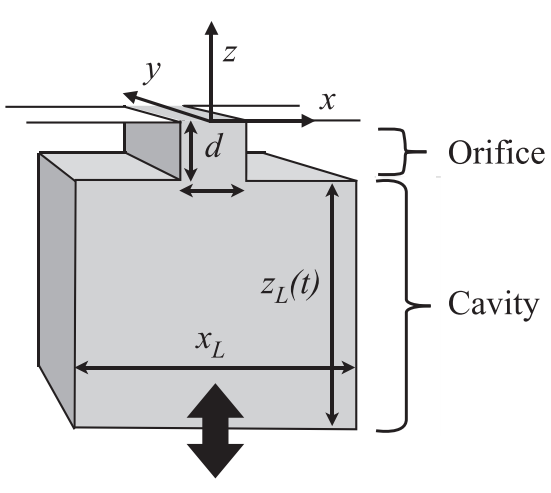

Figure 1 Geometric configuration of synthetic jet

a small amplitude. The sequence of separation control using the synthetic jet can be briefly described as follows. First, the periodic deformation of the cavity produces periodic blowing and suction flow from the orifice; the resulting flow from the orifice fluctuates the separated shear layer near the exit and then, the fluctuation is amplified as it convects downstream. Finally, the entire separated flow is modified and an attached flow is obtained. Note that the momentum flux directly provided by the SJ is so negligible that piezoelectric devices, pistons, and speakers are used to oscillate the cavity bottom [3-5]. Therefore, the SJ is considered suitable for unmanned air vehicles (UAV) and microscale air vehicles (MAV) because of its simple structure and low energy consumption. In addition, many experimental and numerical studies in related literature report that the SJ is applicable for separation control not only over an airfoil but also for other fluidic machineries, e.g., turbine blades.

Recently, three-dimensional (3D) unsteady analyses are getting more and more significant with regards to the physics of the separation control, focusing on turbulent structures that are 3D and unsteady. For example, Okada et al. performed a LES on the separation control over a backward facing step using an SJ with a $3 \mathrm{D}$ cavity [6]. The characteristics of turbulent structures in external flow controlled by the synthetic jet were also discussed. You and Moin also conducted LES [7] on the separation control around an airfoil; the aerodynamic coefficients from the simulation corresponded well to those obtained experimentally. They also reported that the key mechanisms of separation control include not only the modification of two-dimensional (2D) boundary layer profile by adding or removing momentum in the free-stream direction, but also 3D turbulent mixing. However, they did not examine control effects on actuation frequency and a quantitative discussion has never been conducted in terms of the relationship between turbulent mixing and separation control over an airfoil.

In recent studies, the computation of unsteady flows using high-resolution schemes in an external flow as well as in a deforming cavity is regarded as significant. In this study, the simulation of separation control using an SJ is conducted around an NACA 0015 airfoil by LES with a compact scheme [8]. The control effects of the actuation frequency $F^{+}$, which is a normalized frequency of SJ based on chord length and free-stream velocity, are observed. Also, the aim of the present research was to clarify the effects of actuation frequency on separation control and to classify its mechanism, especially in terms of turbulent 
statistics. The relationship between a spatial distribution of Reynolds shear stress and the actuation frequency $F^{+}$is investigated in detail.

\section{PROBLEM SETTINGS}

In this section, the flow condition and configurations of SJ are presented. Note that further, the asterisk denotes the dimensional value; the terms "leading edge" and "trailing edge" are frequently used which indicate the location of $0 \%$ and $100 \%$ of the airfoil chord.

\subsection{Flow Conditions}

The flow is around the NACA 0015 airfoil and the angle of attack is set to $12^{\circ}$. The free-stream Mach number is set to 0.2 as the compressibility of the fluid is almost negligible except near the leading edge. The Reynolds number, defined by the chord length $c_{h}^{*}$ and freestream velocity $u_{\infty}^{*}$, is set to 63,000 . The flow is completely separated from $2.5 \%$ of the airfoil chord without control. The fluid is assumed to be air and the specific heat ratio and the Prandtl number are set to 1.4 and 0.72 , respectively.

\subsection{Geometric Configuration and Control Variables of Synthetic Jet}

In this section, the configuration of SJ is described. The SJ is installed at the leading edge $\left(x / c_{h}=0.0\right)$ of the airfoil, and its orifice is normal to the airfoil surface. In this study, the leading edge is adopted as the position of the SJ, which has been reported effective for a wide variety of airfoils [3,9]. In the following, all nondimensional values are based on the free-stream velocity $u_{\infty}^{*}$, the wing chord length $c_{h}^{*}$, and the freestream density $\rho_{\infty}^{*}$; they are denoted without a superscript “*," e.g., pressure $p$, density $\rho$, and velocity vector $\boldsymbol{u}=(u, v, w)$. The nondimesional value of actuation frequency $f^{*}$ is denoted by $F^{+}$according to the previous studies $[3,6,10]$ which is defined as $F^{+} \equiv f^{*} c_{h}^{*} / u_{\infty}^{*}$. Note that $c_{h} \equiv 1$ and $u_{\infty} \equiv 1$ hold according to the definition above but $c_{h}$ and $u_{\infty}$ are often explicitly written for ease of understanding below. For simplicity, 2D shapes along the span are adopted for the orifice and cavity, as shown in Fig.1. The orifice width is set to $0.5 \%$ of the chord length $\left(d=0.005 c_{h}\right)$, which was often used in previous studies $[7,11]$; the orifice height is set to $d$; the cavity depth is set as $z_{L 0}=10 d$; and the cavity bottom width is set as $x_{L}=5.5 d$. The shapes of the cavity and orifice are similar to those used in the previous study [11]. The bottom of the cavity oscillates in a translational motion:

$$
z_{L}(t)=z_{L 0}+A \cos \left(2 \pi F^{+} t\right)
$$


where $z_{L}(t)$ is the depth of cavity; $t$ is the time; and $A$ is the amplitude of oscillation.

The momentum coefficient $C_{\mu}$ is defined by:

$$
C_{\mu} \equiv \frac{\rho u_{\max }^{2} d}{\rho u_{\infty}^{2} c_{h}}, \quad u_{\max } d \equiv x_{L} \max \left(\frac{d z_{L}(t)}{d t}\right)=2 \pi x_{L} A F^{+}
$$

which is the ratio of the momentum induced by the SJ and the free stream. Here, the fluid in the cavity is assumed to be incompressible, and the momentum induced by the SJ $\rho u_{\max }$ is determined according to the cavity depth, $z_{L}(t)$. Note that the amplitude $A$ is changed according to $F^{+}$when $C_{\mu}$ is kept constant as

$$
A=\frac{u_{\infty} \sqrt{c_{h} C_{\mu} d}}{2 \pi F^{+} x_{L}} .
$$

\subsection{Computational Cases of Control Variables}

The computational cases are arranged in Table 1. In this study, the momentum coefficient $C_{\mu}$ is kept to $0.2 \%$ which is smaller than that used in the previous study [7].

Table 1 Synthetic jet parameters

\begin{tabular}{ccrc}
\hline Case $^{1}$ & Synthetic jet is installed at & $F^{+}$ & $A$ \\
\hline Control off & - & - & - \\
A & Leading edge & 1 & $0.0180 c_{h}$ \\
B & Leading edge & 6 & $0.0031 c_{h}$ \\
C & Leading edge & 10 & $0.0018 c_{h}$ \\
\hline
\end{tabular}

${ }^{1} C_{\mu}$ is set to 0.002 in all the cases.

\section{COMPUTATIONAL APPROACH}

\subsection{Computational Grids}

Table 2 Grid points

\begin{tabular}{crcrr}
\hline Zone & \multicolumn{1}{c}{$\xi$} & \multicolumn{1}{c}{$\eta$} & \multicolumn{1}{c}{$\zeta$} & All points \\
\hline 1 & 795 & 134 & 179 & $19,068,870$ \\
2 & 253 & 134 & 91 & $3,085,082$ \\
3 & 45 & 134 & 75 & 452,250 \\
4 & 157 & 134 & 214 & $4,502,132$ \\
\hline
\end{tabular}

In this study, a zonal grid approach [12] is employed: background grid around airfoil (zone 1), intermediate region (zone 2), cavity of the synthetic jet (zone 4), and orifice of the SJ (zone 3) are generated separately as shown in Fig. 1. Every fifth or tenth point in each direction is presented in this figure. On the boundaries where the zonal grids are connected with each other, approximately 20 grid points are overlapped and the flow variables are exchanged with small errors [12]. The total number of grid points is approximately 30 million (Table 2). 


\subsubsection{Background grid}

The C-type grid is adopted around the airfoil, and the outer boundary is located at $25 c_{h}$ away from the leading edge. The length of the computational region along the span ( $y$ direction) is $0.2 c_{h}$ and a periodic boundary condition is applied to the spanwise boundaries. The boundary-fitted coordinate system $(\xi, \eta, \zeta)$ is employed for computation; the minimum grid size in the direction normal to the airfoil surface ( $\zeta$ direction) is $0.12 \%$ of the chord length $c_{h}$ (or $0.03 / \sqrt{\mathrm{Re}}$ ).

\subsubsection{Grids around the synthetic jet}

Four grids (zones 1 to 4 ) are employed for the computation (Fig. 2). The minimum size of the orifice and cavity grids (zones 3 and 4) corresponds to that of the background grid (zone 1). The grid system for the cavity region (zone 4) deforms [13] according to the oscillation of the cavity bottom.

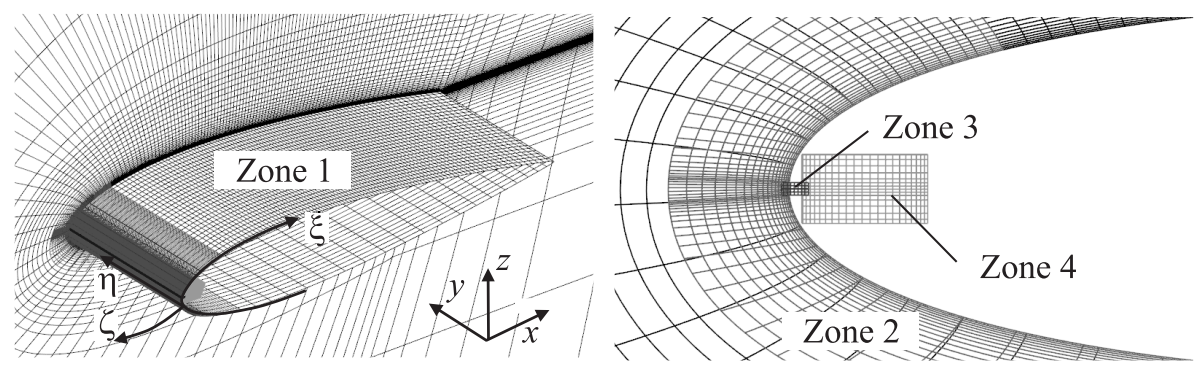

Figure 2 Computational grids

\subsection{Computational Methods}

In this study, LANS3D [14], a fluid analysis solver developed at ISAS/JAXA, is employed for the series of computations. The governing equations are the filtered 3D compressible Navier-Stokes equations without subgrid-scale (SGS) stress and heat flux terms. These equations are solved in body-fitted coordinates $(\xi, \eta, \zeta)$. As the fluid structure in the cavity and orifice is very small and unsteady, a high-resolution unsteady computational method is required. Thus, the spatial derivatives of convective terms and viscous terms, metrics, and the Jacobian are evaluated by a six-order compact difference scheme [8]. Near the boundary, second-order explicit difference schemes are used. Tenth-order filtering [15] is used with a filtering coefficient of 0.495. For time integration, lower-upper symmetric alternating direction implicit and symmetric Gauss-Seidel(ADI-SGS) [16] methods are used. To ensure time accuracy, a backward second-order difference formula is used for time integration and five subiterations [17] are adopted. The 
computational time step is $2 \cdot 10^{-4}$ in nondimensional time to obtain a maximum Courant-Friedrichs-Lewy (CFL) number less than 2 for the controlled cases, which is determined by the previous studies using LES for turbulent boundary layer $[10,18]$. Using these methods, an implicit LES (ILES) approach $[17,19]$ is adopted. In the ILES approach, unlike the standard LES approach, no additional stress and heat flux are appended to the SGS models. Instead, in this study, a high-order, low-pass filter selectively damps only poorly resolved, highfrequency waves. At the outflow boundary, all variables are extrapolated from one point in front of the outflow boundary (pressure is fixed to that of the freestream). On the surface of the airfoil, cavity, and orifice, no-slip conditions are adopted. A periodic boundary condition is applied to the spanwise boundaries. Note that the applicability of the computational code with respect to various flows have been well examined in [20-22]. In addition, on the deforming grid (zone 4), the geometric conservation law (the GCL identity) is satisfied using symmetric conservative metrics proposed by Abe et al. [23].

\subsection{Verification of the Computation}

Table 3 Aerodynamic coefficients

\begin{tabular}{cccc}
\hline Grid density & $C_{L}$ & $C_{D}$ & $C_{L} / C_{D}$ \\
\hline Coarse & 0.410 & 0.143 & 2.85 \\
Medium & 0.458 & 0.157 & 2.92 \\
Fine & 0.477 & 0.159 & 2.99 \\
\hline
\end{tabular}

A series of verification tests for the separated flow around the NACA 0015 airfoil, where the angle of attack is $12^{\circ}$, has been conducted. Three types of computational grids were prepared for verification: coarse, medium, and fine grids in which the number of grid points was 9 million, 19 million, and 44 million, respectively. The medium grid corresponds to the grid presently used in this

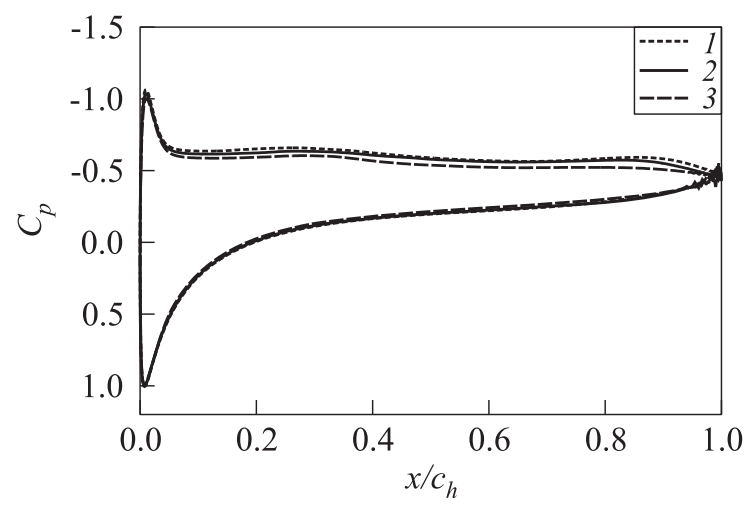

Figure 3 Distribution of time-averaged flow fields $C_{p}$ on fine (1), medium (2), and coarse (3) grids 


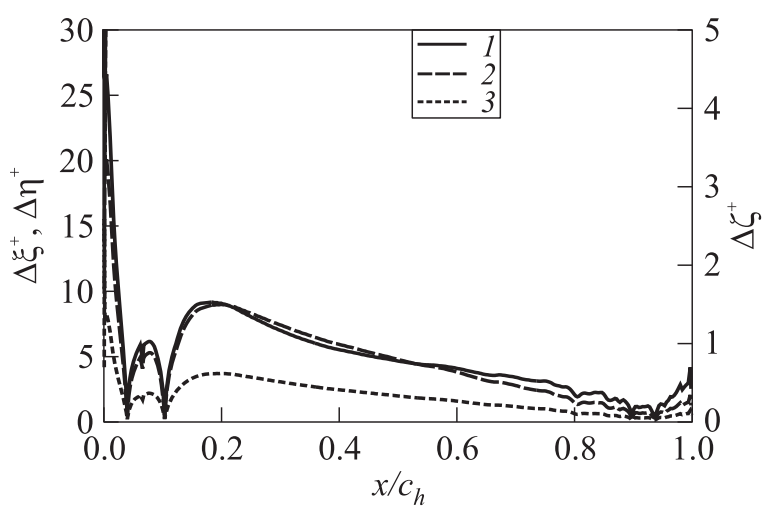

Figure 4 Grid sizes in the wall units near the wing surface for the controlled case $\left(F^{+}=6.0\right): 1-$ chordwise, $\Delta \xi^{+} ; 2-$ spanwise, $\Delta \eta^{+} ;$and $3-$ wall-normal, $\Delta \zeta^{+}$

paper. Table 3 shows the aerodynamic coefficients $\left(C_{L}, C_{D}\right.$, and $\left.C_{L} / C_{D}\right) ;$ both $C_{L}$ and $C_{D}$ slightly increase with the grid density. On the other hand, the suction peak at the leading edge and the plateau pressure distribution are similarly observed for all grids as shown in Fig. 3. In addition, for the separation controlled flow with $F^{+}=6$, the grid sizes in the wall unit were obtained as $\left(\Delta \xi^{+}, \Delta \eta^{+}, \Delta \zeta^{+}\right)$ $\leq(10,9,1)$ in the attached-flow

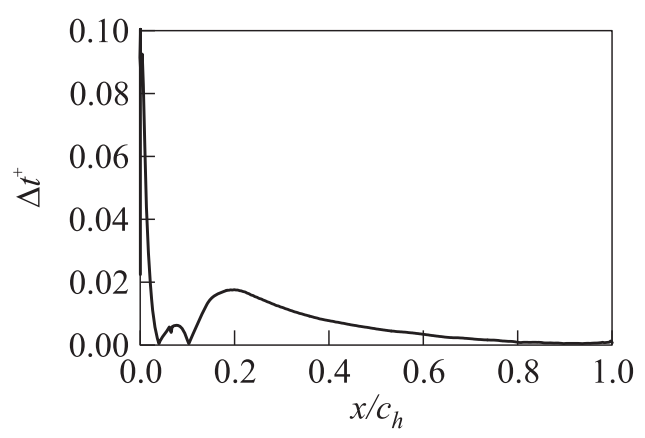

Figure 5 Time-step size in the wall unit for the controlled case $\left(F^{+}=6.0\right)$ region over the airfoil (Fig. 4).

These values satisfy the criteria of grid spacing to resolve a near-wall turbulence proposed by Kawai and Fujii [22] and Teramoto [18]. Therefore, the grid density of the medium grid is sufficient for discussion on the separation control effects in this paper. On the other hand, the computational time-step size in the wall unit was obtained as $\Delta t^{+} \leq 0.1$ in Fig. 5 , which is sufficiently smaller than that proposed by Choi and Moin [24] for turbulent flow. Note that the aerodynamic coefficients of the noncontrolled case have been validated by comparing with those of the experiments [25]. In addition, the Mach number effect for the computation with and without control has been verified using the same grid and scheme although a dielectric barrier discharge (DBD) plasma actuator is employed [26]. The verification of the present GCL scheme was shown in the previous study [23]. 


\section{EFFECT OF ACTUATION FREQUENCY $\boldsymbol{F}^{+}$ ON SEPARATION CONTROL}

\subsection{Aerodynamic Coefficients}

Figure 6 shows the time variance of the lift and drag coefficients $\left(C_{L}\right.$ and $\left.C_{D}\right)$ where the horizontal axis denotes nondimensional time $t u_{\infty} / c_{h}$. In Fig. 6, SJ actuates for $16 \leq t u_{\infty} / c_{h} \leq 28$ and the corresponding sequence is shown. Curve 1 represents the case without actuation, while curves 2,3 , and 4 represent the controlled case with $F^{+}=1,6$, and 10 , respectively. In all controlled cases, the lift coefficient $C_{L}$ increases and the drag coefficient $C_{D}$ reduces. Thus, all controlled cases attain stall recovery. Aerodynamic coefficients oscillate according to the actuation frequency $F^{+}$in each controlled case and a large flow structure is expected to appear periodically on the airfoil.

Table 4 and Fig. $7 a$ show time-averaged aerodynamic coefficients (averaged in $\left.20 \leq t / u_{\infty} c_{h} \leq 28\right)$. The lift-drag coefficient $C_{L} / C_{D}$ is recovered the most at $F^{+}=6$ (see Table 4 ), but the lift coefficient $C_{L}$ is recovered the most

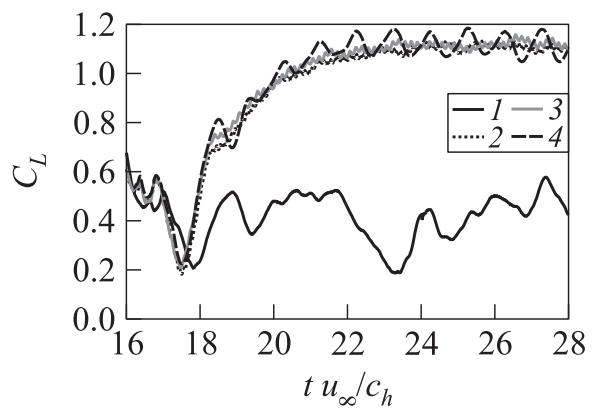

(a)

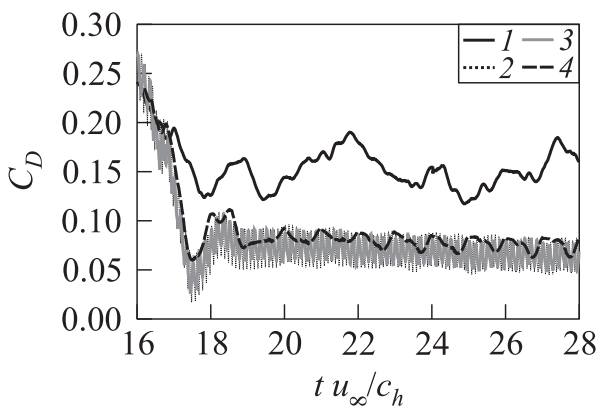

(b)

Figure 6 Time history of aerodynamic coefficients $C_{L}(a)$ and $C_{D}(b): 1-$ control off; $2-F^{+}=1 ; 3-6$; and $4-F^{+}=10$

Table 4 Time-averaged $C_{L}$ and $C_{D}$ values in $20 \leq t u_{\infty} / c_{h} \leq 28$

\begin{tabular}{c|llc}
\hline Case & $C_{L}$ & $C_{D}$ & $C_{L} / C_{D}$ \\
\hline Control off & 0.427 & 0.151 & 2.82 \\
$F^{+}=1$ & 1.10 & 0.0775 & 14.2 \\
$F^{+}=6$ & 1.10 & 0.0669 & 16.4 \\
$F^{+}=10$ & 1.08 & 0.0670 & 16.1 \\
\hline
\end{tabular}




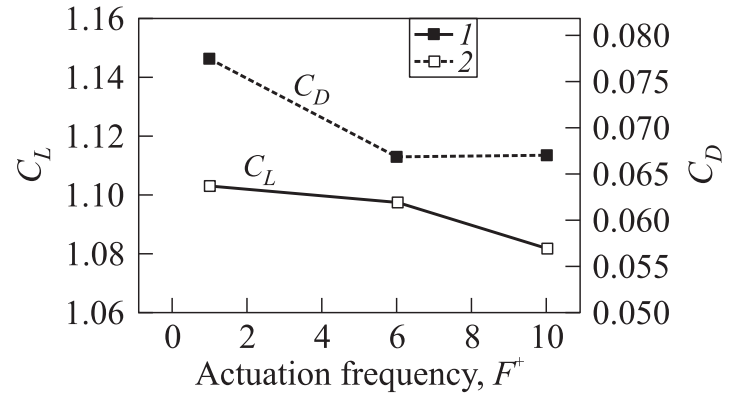

(a)

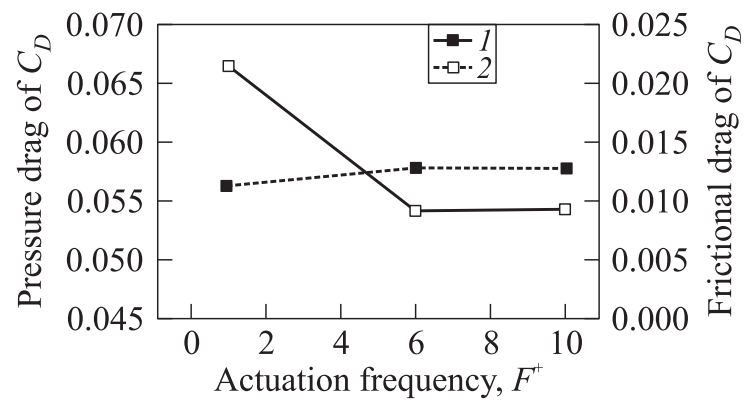

(b)

Figure 7 Time-averaged aerodynamic coefficients: (a) $C_{L}$ and $C_{D}$ values arranged by $F^{+}$; and (b) decomposition of $C_{D}$ : pressure (1) and frictional (2) drags

at $F^{+}=1$ (see Table 4 and Fig. $7 a$ ). Therefore, higher lift-drag coefficient is due to lower drag coefficient in the present flow condition. In particular, the drag coefficient is decomposed into pressure and frictional components in Fig. $7 b$. The horizontal axis is the actuation frequency $F^{+}$, and the left and right vertical axes are the pressure and frictional drags, respectively. Figure $7 b$ shows that the pressure drag is dominant for all controlled cases, and it is the most reduced for $F^{+}=6.0$. In the following subsection, the time-averaged flow fields are discussed in terms of the reduction of pressure drag.

\subsection{Separation Bubble in Time-Averaged Flow Fields}

Here, the time-averaged flow fields of velocity $u$ (in the $x$ direction) and pressure $p$ are arranged for all controlled cases. Figure 8 illustrates the velocity fields $u$, and each flow is attached to the airfoil surface except for the case without control. Each frame includes a zoom view near the leading edge and in all controlled cases, a reversed region (in blue) is observed. The reversed region corresponds to the separation bubble where the spatial distribution of the pressure 


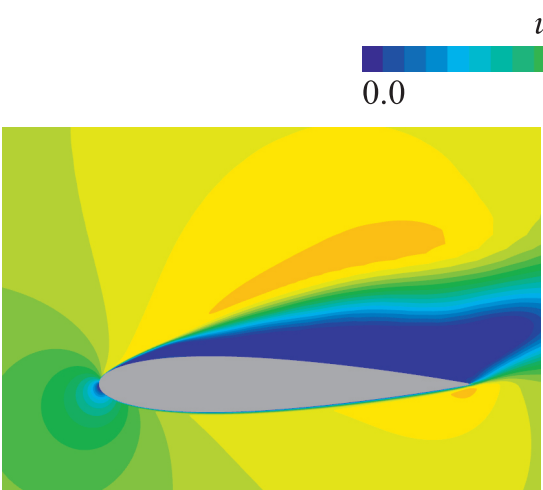

(a)

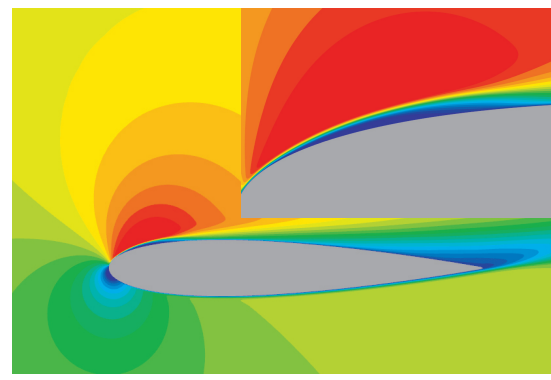

(b)

\section{5}

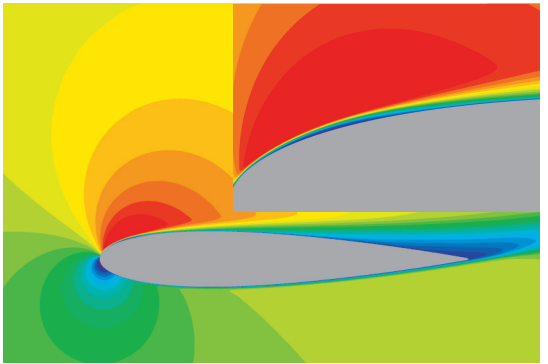

(c)

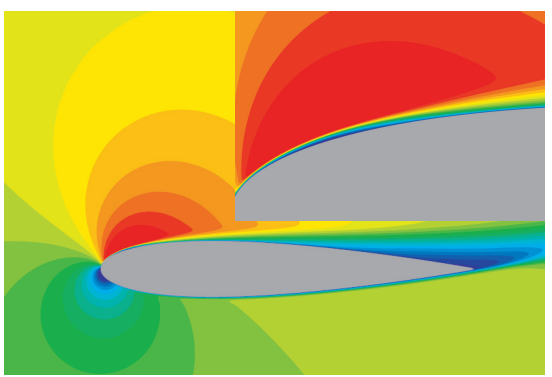

(d)

Figure 8 Time averaged flow field (averaged in $20 \leq t u_{\infty} / c_{h} \leq 28$ ) of the $x$-direction velocity $u / u_{\infty}$ : $(a)$ control off; $(b) F^{+}=1 ;(c) 6$; and $(d) F^{+}=10$

coefficient $C_{p}$ shows a plateau region in Fig. $9 a$. In particular, the separation bubble is the largest for $F^{+}=1$ and it leads to the weakest suction peak at the leading edge. On the other hand, the case with $F^{+}=6$ shows the strongest suction peak, which contributes to a strong thrust at the leading edge as shown in Fig. $9 b$. Note that Fig. $9 b$ shows the $C_{p}$ distribution with respect to the $z_{H}$ component $\left(z_{H}\right.$ axis is inclined by $12^{\circ}$ from the $z$ axis), which directly illustrates the difference of contribution to drag (or thrust) for each $F^{+}$although Fig. $9 a$ shows the contribution to both of lift and drag. Therefore, the lift-drag coefficient is recovered the most for $F^{+}=6$, where the pressure drag is significantly suppressed due to the strong suction peak at the leading edge. Conversely, the friction drag slightly increases with the increasing $F^{+}$in Fig. $7 b$ because the turbulent transition occurs earlier and turbulent boundary layer widely covers the airfoil surface with the increasing $F^{+}$. However, the contribution from the 

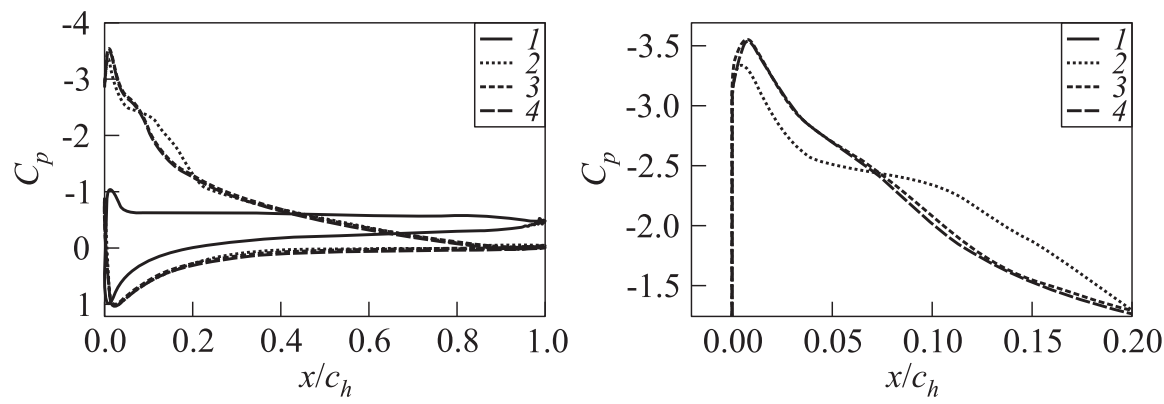

(a)
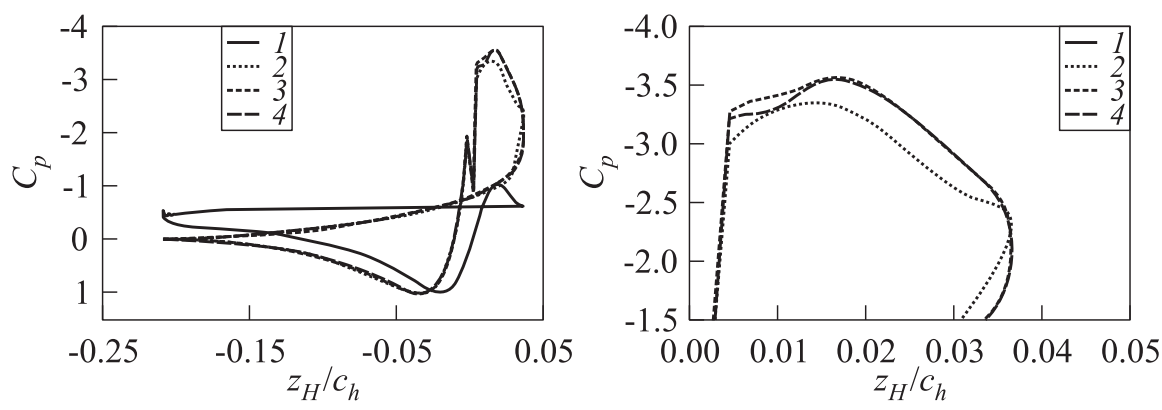

(b)

Figure 9 Distribution of time-averaged flow field $C_{p}$ with respect to $x / c_{h}(a)$ and with respect to $z_{H} / c_{h}$ ( $z_{H}$ axis is inclined by $12^{\circ}$ from $z$ axis) $(b): 1$ - control off; $2-F^{+}=1 ; 3-6$; and $4-F^{+}=10$

friction-drag increase to the total-drag variance is less than $5 \%$ which does not determine the trend of aerodynamic coefficients in the present flow condition. On the other hand, the case with $F^{+}=10$ attains lower lift-drag ratio than $F^{+}=6$. The reason is that the separation bubble size is slightly smaller when $F^{+}=10$ than 6 , which reduces the lift coefficient while the pressure drag remains almost the same for $F^{+}=10$ and 6 . In the following subsections, two cases with $F^{+}=1$ and 6 are examined because the size of the separation bubble and resulting performance is significantly different between these cases.

\subsection{Instantaneous Flow Fields and Turbulent Transition}

Figure 10 shows the instantaneous flow fields of the controlled cases with $F^{+}$ $=1.0$ and 6.0. The isosurface near the airfoil surface is the second invariant of the velocity gradient tensor and is colored by the vorticity in the chord direction ( $x$-axis). The contour plane normal to the span direction ( $y$-axis) shows the $x$ component of the velocity $u / u_{\infty}$. In both controlled cases, 3D fine vortex struc- 


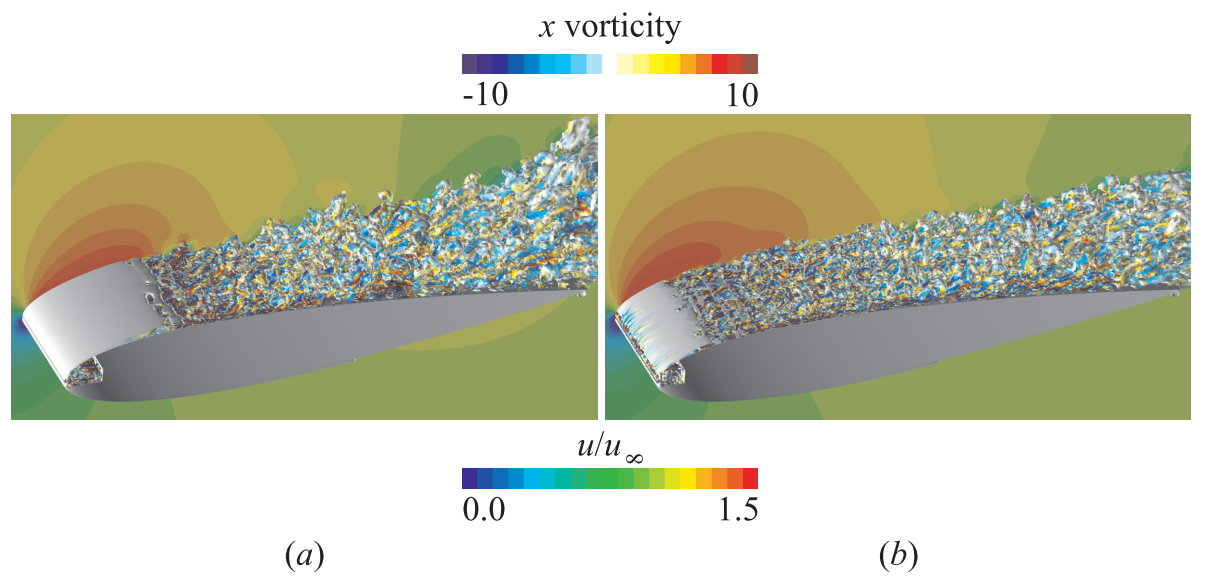

Figure 10 Instantaneous flow field: $x$-direction velocity contour and isosurfaces of the second invariant of velocity gradient tensor (colored by $x$ vorticity): $(a) F^{+}=1$; and $(b) F^{+}=6$

tures are developed over the airfoil, and a turbulent boundary layer is observed. On the other hand, a 2D separated shear layer appears near the leading edge in both snapshots, but it expands more in the downstream direction for $F^{+}=1$ than 6 . The difference in separated shear layers leads to the difference in the size of the separation bubble in the time-averaged field shown in Fig. 8. Note that the snapshots in Fig. 10 are taken when a suction phase of SJ has been achieved. The unsteady behavior of the separation bubble will be discussed later using phase-averaged flows based on $F^{+}$.

Figure 11 shows the spatial distribution of a velocity fluctuation $\left(u^{\prime}, v^{\prime}, w^{\prime}\right)$ with the reverse velocity region. This region corresponds to the location of the separation bubble in Fig. 8 and each component of the velocity fluctuation shows the maximum at the end of the separation bubbles. More precisely, the peak of $v^{\prime}$ is located further downstream than those of $u^{\prime}$ and $w^{\prime}$ for $F^{+}=6$. This trend is typically observed on a laminar separation bubble [27] where the turbulent transition occurs.

Figure 12 shows the power spectra of $u$ in $x / c_{h}=0$ to 0.2 . Note that along a certain grid line, the turbulent kinetic energy takes its maximum value on each sampling point. In all cases, the power spectral density (PSD) of high frequencies $(\simeq 100)$ almost follow the straight line which indicates the Kolmogorov's 5/3 law and the well-developed turbulent boundary layer is observed. On the other hand, the peaks which correspond to the actuation frequency $F^{+}$and its harmonic component remain even at $20 \%$ of the chord length. This indicates that a large flow structure periodically appears on the airfoil surface whose period is the same as $F^{+}$and it affects the fluctuation of the aerodynamic coefficient in Fig. 6 . 


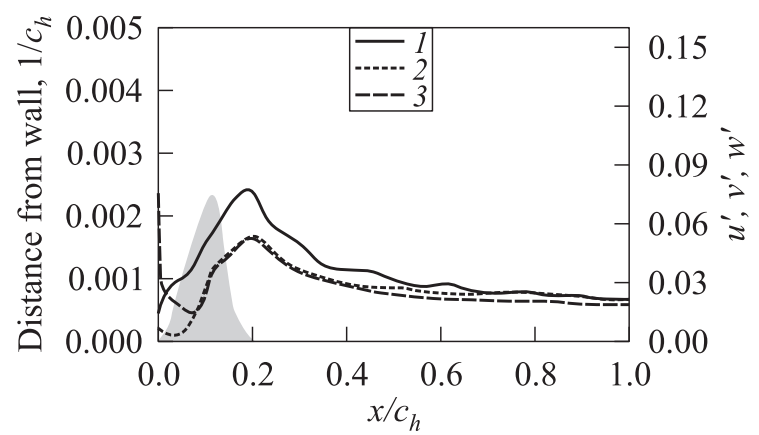

(a)

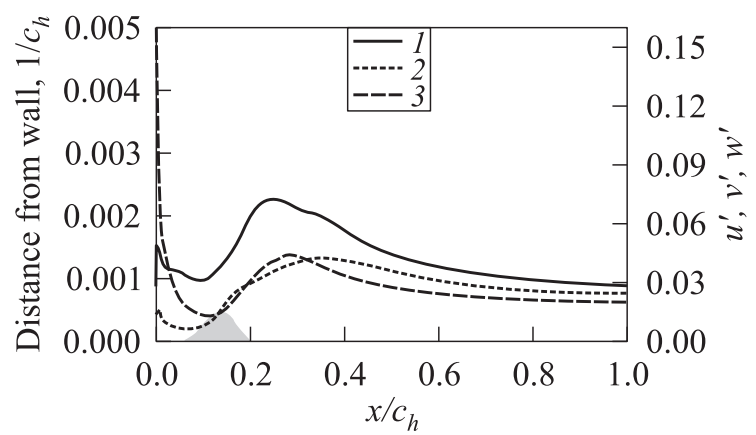

(b)

Figure 11 Spatial distribution of a fluctuation of flow velocity: $1-u^{\prime} ; 2-v^{\prime}$; and $3-w^{\prime}$. The grey colored region with the left axis shows the reversed flow velocity region (SJ at 0\%): $(a) F^{+}=1$; and $(b) F^{+}=6$

\section{MECHANISM OF SEPARATION CONTROL FOCUSING ON TURBULENT MIXING}

In this section, the relationship between turbulent mixing and large flow structure is investigated. The turbulent mixing is quantitatively examined using the $-\overline{u^{\prime} w^{\prime}}$ component of a Reynolds shear stress, which corresponds to an induction of momentum from the free stream.

On the other hand, in the previous section, it has been suggested that a large flow structure periodically appears on the airfoil.

Thus, a phase-averaging procedure was conducted to extract the periodic large flow structures whose period corresponds to the actuation frequency $F^{+}$. For this purpose, the instantaneous physical quantity $f=f(t, x, y, z)$ was decomposed into overall average, $\bar{f}$, phase fluctuation, $\tilde{f}$, and turbulent fluctuation, $f^{\prime \prime}$, as follows: 


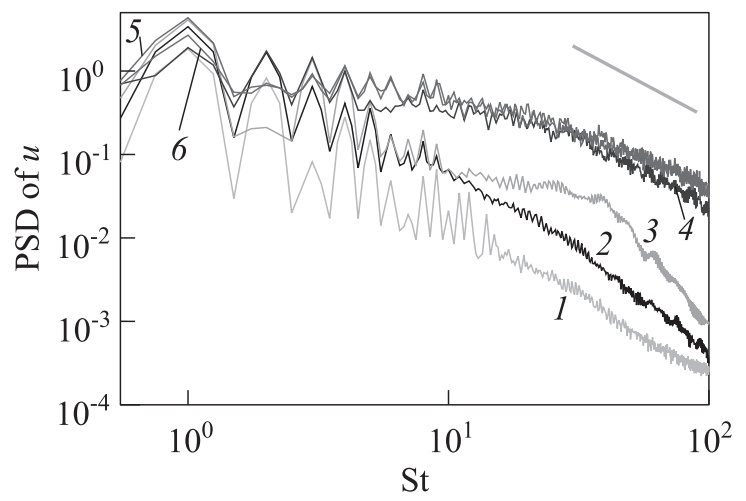

(a)

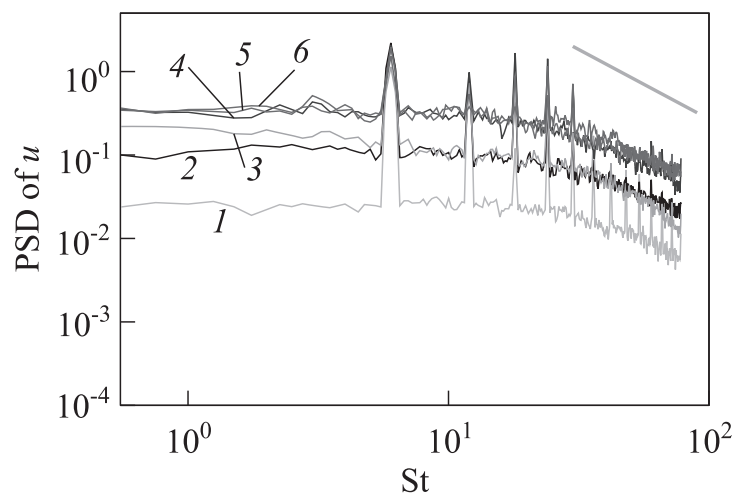

(b)

Figure 12 Power spectra of $u$ component of all controlled cases; the horizontal axis is a frequency normalized by freestream flow velocity and chord length: $1-0.0 \%$ of chord length; $2-2.5 \%$ of chord length; $3-5.0 \%$ of chord length; $4-10 \%$ of chord length; $5-15 \%$ of chord length; and $6-20 \%$ of chord length. The straight grey line shows Kolmogorov's 5/3 law: $(a) F^{+}=1$; and $(b) F^{+}=6$

$$
\begin{aligned}
f(t, x, y, z) & =\underbrace{\bar{f}(x, y, z)}_{\text {overall average }}+\underbrace{f^{\prime}(t, x, y, z)}_{\text {overall fluctuation }} \\
& =\underbrace{\bar{f}(x, y, z)}_{\text {overall average }}+\underbrace{\tilde{f}(\varphi, x, y, z)}_{\text {phase fluctuation }}+\underbrace{f^{\prime \prime}(t, x, y, z)}_{\text {turbulent fluctuation }} \\
& =\underbrace{\langle f\rangle(\varphi, x, y, z)}_{\text {phase average }}+\underbrace{f^{\prime \prime}(t, x, y, z)}_{\text {turbulent fluctuation }}
\end{aligned}
$$

where $\varphi$ indicates the phase angle. In this study, the phase-averaging procedure (i.e., computing $\langle f\rangle$ ) is based on the actuation frequency $F^{+}$and is approxi- 
mated by dividing the acutuation period into 10 segments $(\varphi=2 \pi / 10,4 \pi / 10, \ldots$. $\ldots, 20 \pi / 10)$. According to Eq. (1), the Reynolds shear stress, $\overline{u^{\prime} w^{\prime}}$, can be decomposed into overall phase component, $\overline{\tilde{u} \tilde{w}}$, and overall turbulent component, $\overline{u^{\prime \prime} w^{\prime \prime}}$, as follows:

$$
\overline{u^{\prime} w^{\prime}}(x, y, z)=\overline{\tilde{u} \tilde{w}}(x, y, z)+\overline{u^{\prime \prime} w^{\prime \prime}}(x, y, z) .
$$

On the other hand, the phase-averaged Reynolds shear stress, $\left\langle u^{\prime} w^{\prime}\right\rangle$, is decomposed into phase component, $\langle\tilde{u} \tilde{w}\rangle$, and turbulent component, $\left\langle u^{\prime \prime} w^{\prime \prime}\right\rangle$, as follows:

$$
\left\langle u^{\prime} w^{\prime}\right\rangle(\varphi, x, y, z)=\langle\tilde{u} \tilde{w}\rangle(\varphi, x, y, z)+\left\langle u^{\prime \prime} w^{\prime \prime}\right\rangle(\varphi, x, y, z) .
$$

After the phase-averaging procedure, the decomposition given by Eq.(2) is discussed in subsection 5.1, and the decomposition at each phase given by Eq.(3) is focused in subsection 5.2.

\section{Reynolds shear stress \\ $-0.011$ \\ 0.011}

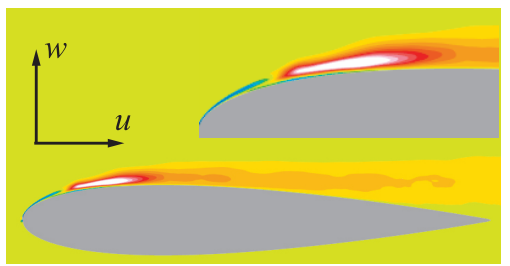

II

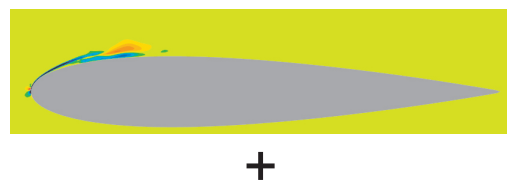

(b)

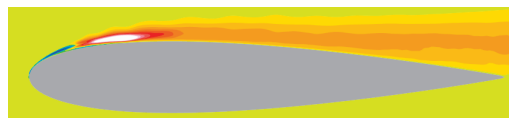

(a)

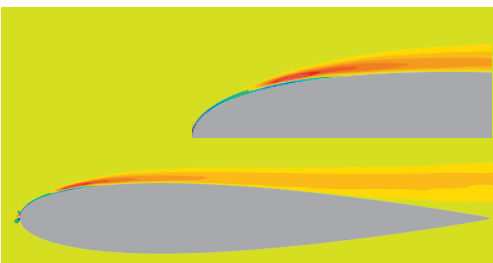

II
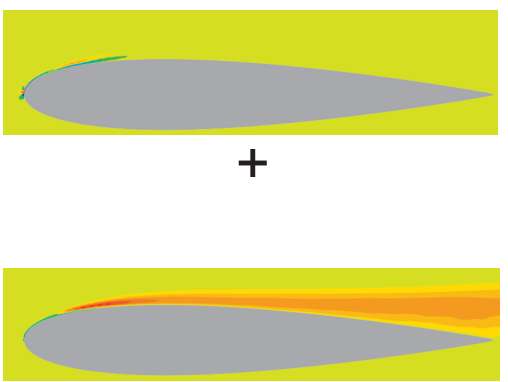

(c)

Figure 13 Spatial distribution of Reynolds shear stress $-\overline{u^{\prime} w^{\prime}} / u_{\infty}^{2}(a)$. Reynolds shear stress is decomposed into periodic $-\overline{\tilde{u} \tilde{w}} / u_{\infty}^{2}(b)$ and turbulent (nonperiodic) $-u^{\prime \prime} w^{\prime \prime} / u_{\infty}^{2}(c)$ components: left column $-F^{+}=1$; and right column $-F^{+}=6$ 


\subsection{Periodic and Turbulent Components of the Reynolds Shear Stress}

Figure 13 shows the periodic and turbulent (nonperiodic) decomposition of the Reynolds shear stress, $-\overline{u^{\prime} w^{\prime}}$. The decomposition is based on the phase-averaging procedure explained in Eq. (2). The turbulent component is much larger than the periodic component in both controlled cases and the peak is located near the end of the separation bubble where the turbulent transition occurs. In particular, the spatial distribution of turbulent component is similar to that of the total component. These results indicate that the turbulent structure dominates the Reynolds shear stress, $-\overline{u^{\prime} w^{\prime}}$, in both cases. The periodic component is locally enhanced near the end of the separation bubble, which is due to the oscillation of the separation bubble at the actuation frequency $\mathrm{F}^{+}$.

\subsection{Large Flow Structures and Reynolds Shear Stress}

To detect the large flow structure, the span- and phase-averaging procedure is conducted and each flow field is presented at $\varphi=2 \pi / 10,10 \pi / 10$, and $18 \pi / 10$ as shown in Figs. 14 and 15. Note that for each phase, the phase decomposition is first conducted by phase-averaging procedure given by Eq. (3); then, the resultant periodic and turbulent components are simply averaged using all gridpoint values in spanwise direction, and the required span- and phase-averaged flow fields are obtained. The left and middle columns show the decomposition of the Reynolds shear stress, $-\left\langle u^{\prime} w^{\prime}\right\rangle$, where the upper and lower figures show the turbulent and periodic components, respectively. The black contour lines indicate the second invariant of the velocity gradient tensor, which corresponds to a large vortex structure with its axis along the span. The right figures are the instantaneous flow fields, and SJ is in the blowing and suction phases for $2 \pi / 10 \leq \varphi \leq 10 \pi / 10$ and $10 \pi / 10 \leq \varphi \leq 20 \pi / 10$, respectively.

For $F^{+}=1$ (see Fig. 14), the separation bubble near the leading edge is divided into multiple vortex structures due to the disturbance from SJ (see Vor1 and Vor2 at $\varphi=10 \pi / 10$ in Fig. 14b). The current phase averaging procedure (10 segments for a period) shows that only two structures are emitted from the separation bubble. In the fine phase averaging procedure $(60$ segments for a period), many fine vortex structures are emitted and merged together, and the resulting large vortex structures (Vor1 and Vor2) are obtained. Vor1 convects in the downstream direction and is emitted from the trailing edge of the airfoil at the actuation frequency $F^{+}=1$. For $F^{+}=6$ (see Fig. 15), three vortex structures (Vor3, Vor2, and Vor3) are generated from the separation bubble at the phase $\varphi=2 \pi / 10$, and convecting downstream. These three vortex structures merge together at $35 \%$ of the chord length (this event is indicated by black dotted circle at $\varphi=10 \pi / 10$ ), and the resulting large vortex is emitted from 


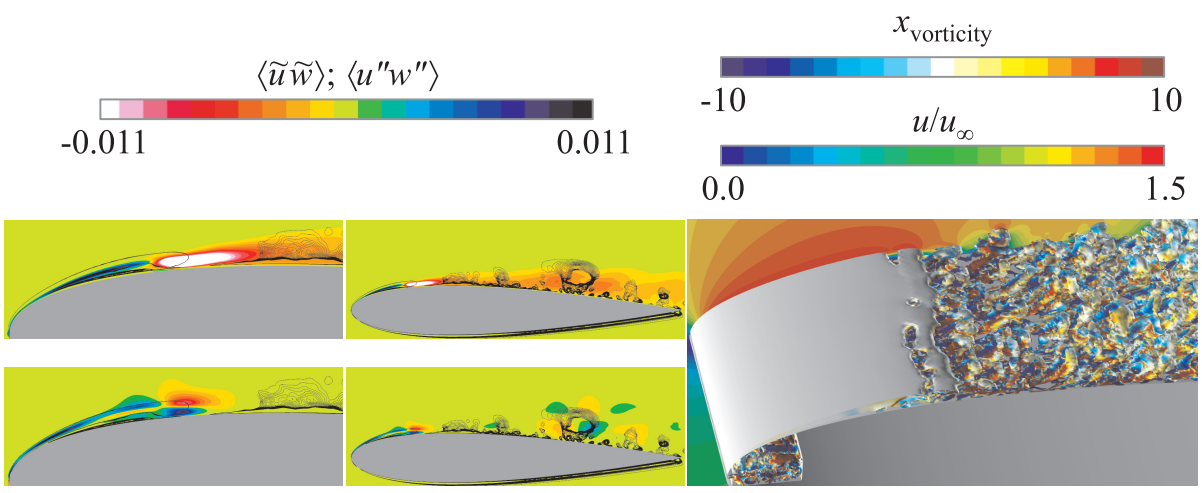

(a)

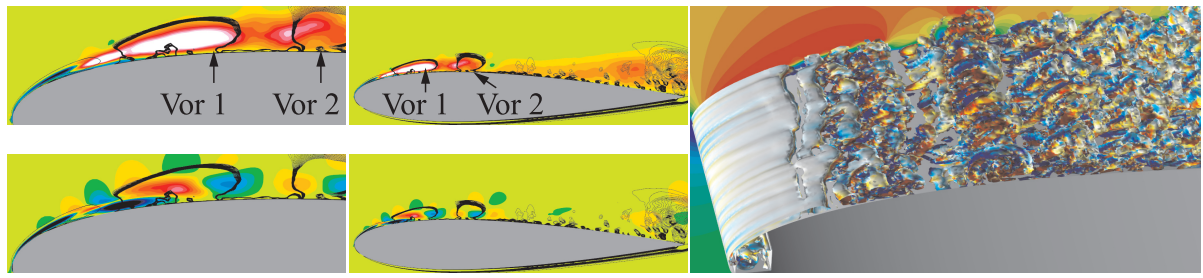

(b)

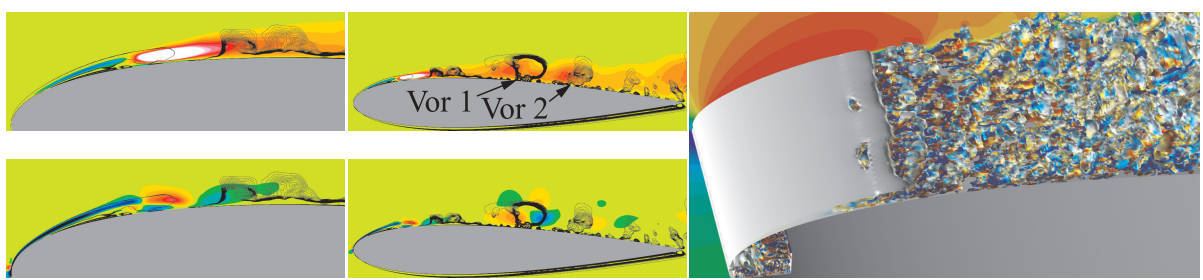

Figure 14 Phase history of periodic, $-\langle\tilde{u} \tilde{w}\rangle$, and turbulent, $-\left\langle u^{\prime \prime} w^{\prime \prime}\right\rangle$, components of Reynolds shear stress, $-\left\langle u^{\prime} w^{\prime}\right\rangle$, in $\varphi=2 \pi / 10(a), 10 \pi / 10(b)$, and $20 \pi / 10(c)$ at $F^{+}=1$. The black contours show the second invariant of the velocity gradient tensor. The black arrows indicate the vortex structures

the trailing edge. The multiple vortex structures include the vortex that was generated in a previous period for $F^{+}=6$. In this way, the detailed structure of the vortices is different between $F^{+}=1$ and 6 , but the resulting large vortex structure convects and is emitted from the trailing edge with the period of $F^{+}$ in both cases. The convection of this large vortex structure is strongly related to the time variance of aerodynamic coefficients shown in Fig. 6. Note that in this paper, the vortex structures (e.g., Vor1) are extracted qualitatively; on the other hand, the present authors also confirmed that these structures can be 


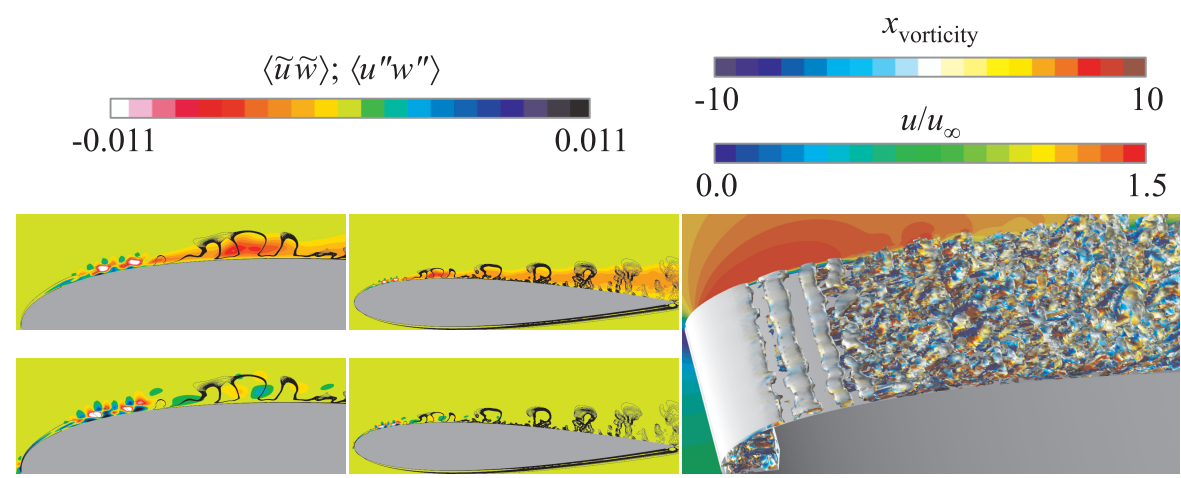

(a)

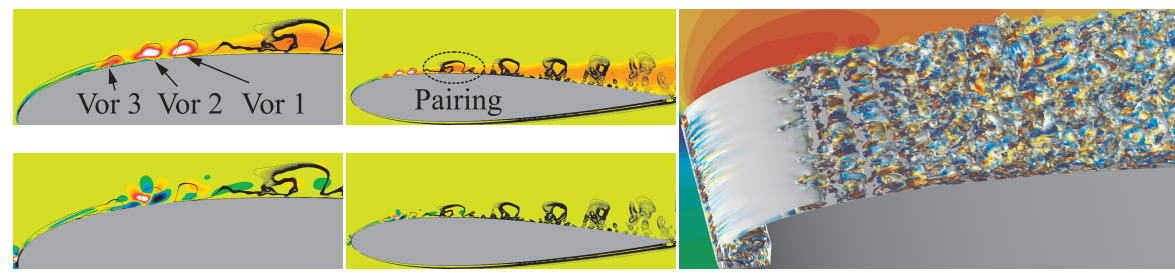

(b)

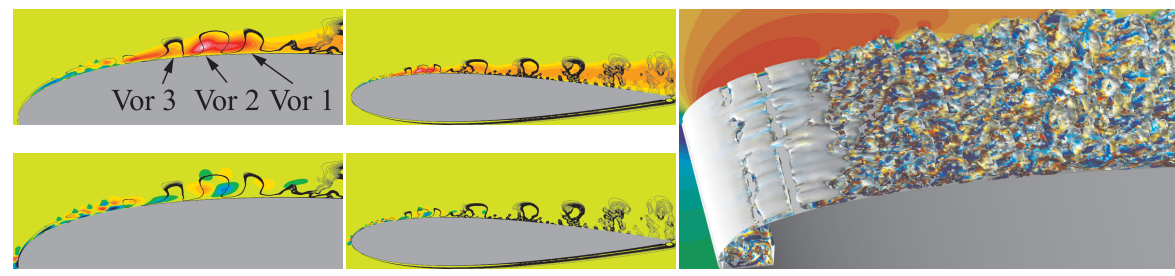

(c)

Figure 15 Phase history of periodic, $-\langle\tilde{u} \tilde{w}\rangle$, and turbulent, $-\left\langle u^{\prime \prime} w^{\prime \prime}\right\rangle$, components of Reynolds shear stress, $-\left\langle u^{\prime} w^{\prime}\right\rangle$, in $\varphi=2 \pi / 10(a), 10 \pi / 10(b)$, and $20 \pi / 10(c)$ at $F^{+}=6$. The black contours show the second invariant of the velocity gradient tensor. The black arrows indicate the vortex structures and black dotted circle indicates the event where those structures merge together

similarly detected using the maximum value of second invariant of the velocity gradient tensor. In both cases, $F^{+}=1$ and 6 , the periodic component of the Reynolds shear stress is locally enhanced at the end of the separation bubble, and the component is convecting in the downstream direction with the large vortex. Thus, the periodic component of the Reynolds shear stress is mainly due to an entrainment by the $2 \mathrm{D}$ motion of the large vortex structure. The turbulent component is more enhanced than the periodic component almost all over the 


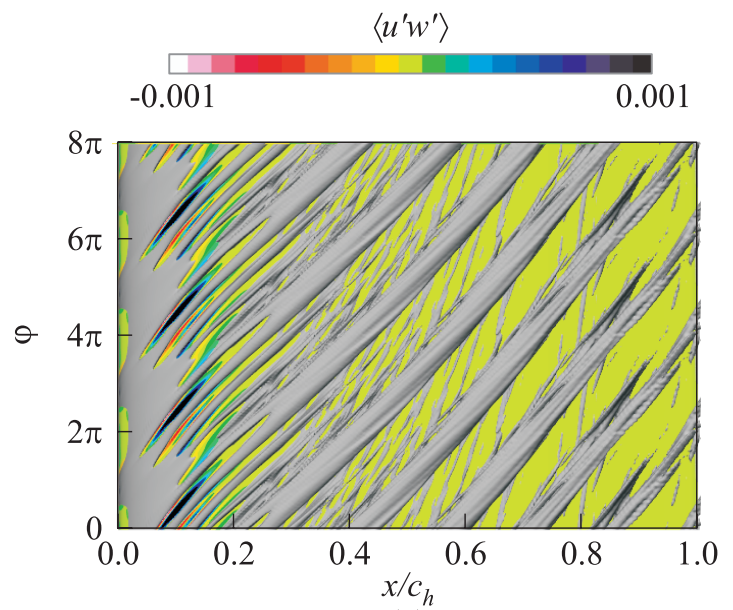

(a)

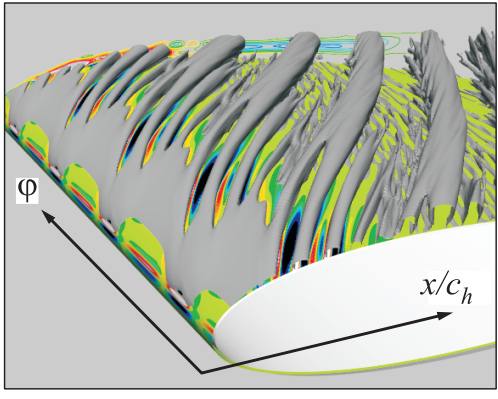

(b)

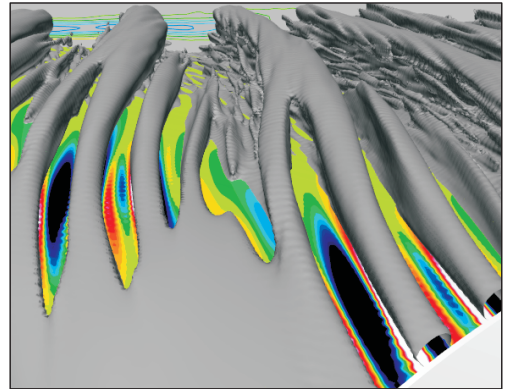

(c)

Figure 16 Phase- and span-averaged flow fields in a combined time-space system. Isosurface is the second invariant of the velocity gradient tensor, and the contour is colored by Reynolds shear stress $-\left\langle u^{\prime} w^{\prime}\right\rangle$

airfoil; therefore, 3D fine structures are dominant in terms of the momentum induction. The turbulent component is also enhanced around the large vortex structure. Therefore, the large vortex structure entrains not only the periodic component but also the turbulent component which is generated by $3 \mathrm{D}$ fine structures. Note that a qualitatively similar trend is observed for $F^{+}=10$.

Finally, the large vortex structures in phase- and span-averaged fields of $F^{+}$ $=6$ are visualized in a combined time space system, which clearly illustrates the merging procedure of two vortices and the convection of the resulting large vortex structure. In Fig. 16, the $\varphi$-axis indicates the phase based on $F^{+}=6$ for $0 \leq \varphi \leq 8 \pi$, and the $x / c_{h}$-axis indicates the spatial chordwise direction. At each phase, the phase- and span-averaged flow fields are visualized, where 
the second invariant of the velocity gradient tensor is shown as gray colored isosurface. Therefore, the isosurface in Fig. 16 shows the convection of large vortex structures which are illustrated by black contours at each phase in Figs. 14 and 15. The contour is colored by the Reynolds shear stress, $-\left\langle u^{\prime} w^{\prime}\right\rangle$. In Fig. $16 a$, it is clearly observed that the separation bubble expands at $\varphi \simeq \pi$ and divides into multiple fine vortex structures at $\varphi \simeq 2 \pi$. In Figs. $16 b$ and $16 c$, the multiple vortex structures merge at the phase $\varphi \simeq \pi$ and convect in the downstream direction with the period of $F^{+}=6.0$.

\section{CONCLUDING REMARKS}

The high-fidelity computations of the separation control using SJ have been conducted over an NACA 0015 airfoil with the angle of attack set to $12^{\circ}$. Synthetic jet is modeled by the deforming cavity and installed at the leading edge of the airfoil. The effects of separation control have been observed at actuation frequencies $F^{+}=1,6$, and 10. Although the time-averaged lift coefficient recovered the most at $F^{+}=1$, lift-drag coefficient became worst at $F^{+}=1$ and recovered the most at $F^{+}=6$ because the pressure drag was significantly suppressed at $F^{+}=6$. The size of the separation bubble near the leading edge is significantly smaller at $F^{+}=6$ than at $F^{+}=1$, and the suction peak of $C_{p}$ is higher at $F^{+}=6$ than at $F^{+}=1$. Such a high suction peak of $C_{p}$ contributes to the reduction of pressure drag at $F^{+}=6$. On the other hand, the case with $F^{+}=10$ shows lower lift-drag coefficient than $F^{+}=6$ because the separation bubble size is slightly smaller at $F^{+}=10$ than 6 , which reduces the lift coefficient while the pressure drag remains almost the same for $F^{+}=10$ and 6 . The friction drag slightly increases with $F^{+}$increasing because the turbulent transition occurs earlier and turbulent boundary layer widely covers the airfoil surface, but in the present flow condition, its contribution to the total drag is too small to affect the trend of aerodynamic coefficients for $F^{+}$.

On the other hand, for all the controlled cases, the periodic flow structures whose period is the same as $F^{+}$have been commonly indicated by the PSD of the chordwise velocity. Therefore, the Reynolds shear stress is decomposed into periodic and turbulent components based on $F^{+}$, and it has been shown that in both cases, the turbulent component is dominant. Meanwhile, large vortex structures have been detected by the phase-averaging procedure based on $F^{+}$ which convect in the downstream direction with the period of $F^{+}$. The turbulent component of the Reynolds shear stress was not uniformly distributed but locally enhanced around the large vortex structure. Therefore, under the present flow conditions, it is concluded that the turbulent structure is significant in terms of the momentum exchange based on Reynolds shear stress and that the large vortex structure specifically entrains the turbulent structure and enhances the turbulent component of Reynolds shear stress. In this way, the trend in the rela- 
tionship between Reynolds shear stress and large vortex structures was similarly observed in the controlled flow fields regardless of the actuation frequency $F^{+}$. Note that the highest aerodynamic performance using $F^{+}=6$ is based on the early and smooth turbulent transition near the leading edge, which would be validated by the most instable mode of spatially developing disturbance in a shear layer (i. e., Kelvin-Helmholtz instability) [25].

\section{ACKNOWLEDGMENTS}

This work was supported by Grant-in-Aid for JSPS Fellows (Grant No. 258793).

\section{REFERENCES}

1. Corke, T. C., C. L. Enloe, and S. P. Wilkinson. 2010. Dielectric barrier discharge plasma actuators for flow control. Annu. Rev. Fluid Mech. 42:505-529.

2. Sato, M., K. Okada, T. Nonomura, H. Aono, A. Yakeno, K. Asada, Y. Abe, and K. Fujii. 2013. Massive parametric study by LES on separated-flow control around airfoil using DBD plasma actuator at Reynolds number 63,000. AIAA Paper No. 2013-2750.

3. Greenblatt, D., and, I. J. Wygnanski. 2000. The control of flow separation by periodic excitation. Prog. Aerosp. Sci. 36:487-545.

4. Amitay, M., D. R. Smith, V.K.D.E. Parekh, and A. Glezer. 2001. Aerodynamic flow control over an unconventional airfoil using synthetic jet actuators. AIAA J. 39(3):361-370.

5. Glezer, A., and M. Amitay. 2002. Synthetic jets. Annu. Rev. Fluid Mech. 34:503529.

6. Okada, K., A. Oyama, K. Fujii, and K. Miyaji. 2010. Computational study on effect of synthetic jet design parameters. Int. J. Aerosp. Eng. 364859. doi: $10.1155 / 2010 / 364959$.

7. You, D., and P. Moin. 2008. Active control of flow separation over an airfoil using synthetic jets. J. Fluid. Struct. 24:1349-1357.

8. Lele, S. K. 1992. Compact finite difference schemes with spectral-like resolution. J. Comput. Phys. 103(1):16-42.

9. Amitay, M. and A. Glezer. 2002. Role of actuation frequency in controlled flow reattachment over a stalled airfoil. AIAA J. 40(2):209-216.

10. Okada, K., T. Nonomura, K. Fujii, and K. Miyaji. 2012. Computational analysis of vortex structures induced by a synthetic jet to control separated flows. Int. J. Flow Control 4(1-2):47-65.

11. Okada, K., A. Oyama, K. Fujii, and K. Miyaji. 2012. Computational study of effects of nondimensional parameters on synthetic jets. Trans. Jpn. Soc. Aeronaut. Space Sci. 55(1):1-11.

12. Fujii, K. 1995. Unified zonal method based on the fortified solution algorithm. J. Comput. Phys. 118:92-108. 
13. Melville, R. B., S. A. Moiton, and D. P. Rizzetta. 1997. Implementation of a fullyimplicit, aeroelastic Navier-Stokes solver. AIAA Paper No. 1997-2039.

14. Fujii, K. 1990. Developing an accurate and efficient method for compressible flow simulations - example of CFD in aeronautics. 5th Conference (International) on Numerical Ship Hydrodynamics Proceedings.

15. Gaitonde, D. V., and M. R. Visbal. 2000. Padé-type higher-order boundary filters for the Navier-Stokes equations. AIAA J. 38(11):2103-2112.

16. Nishida, H., and T. Nonomura. 2009. ADI-SGS scheme on ideal magnetohydrodynamics. J. Comput. Phys. 228:3182-3188.

17. Visbal, M. R., and D. P. Rizzetta. 2002. Large-eddy simulation on general geometries using compact differencing and filtering schemes. AIAA Paper No. 2002-288.

18. Teramoto, S. 2005. Large-eddy simulation of transitional boundary layer with impinging shock wave. AIAA J. 43(11):2354-2363.

19. Visbal, M. R., P.E. Morgan, and D. P. Rizzetta. 2003. An implicit LES approach based on high-order compact differencing and filtering schemes. AIAA Paper No. 2003-4098.

20. Arasawa, T., K. Fujii, and K. Miyaji. 2004. High-order compact difference scheme applied to double-delta wing vortical flows. J. Aircraft 41(4):953-957.

21. Kawai, S., and K. Fujii. 2005. Analysis and prediction of thin-airfoil stall phenomena using hybrid turbulent methodology. AIAA J. 43(5):953-961.

22. Kawai, S., and K. Fujii. 2008. Compact scheme with filtering for large-eddy simulation of transitional boundary layer. AIAA J. 46(3):690-700.

23. Abe, Y., N. Iizuka, T. Nonomura, and K. Fujii. 2013. Conservative metric evaluation for high-order finite difference schemes with the GCL identities on moving and deforming grids. J. Comput. Phys. 232(1):14-21.

24. Choi, H., and P. Moin. 1994. Effects of the computational time step on numerical solutions of turbulent flow. J. Comput. Phys. 113:1-4.

25. Nonomura, T., H. Aono, M. Sato, A. Yakeno, K. Okada, Y. Abe, and K. Fujii. 2013. Control mechanism of plasma actuator for separated flow around NACA0015 at Reynolds number 63,000 - separation bubble related mechanisms. AIAA Paper No. 2013-0853.

26. Asada, K. 2014. Computational analysis of the flow fields induced by a DBD plasma actuator toward separated-flow control. The University of Tokyo. Ph.D. Thesis.

27. Rinoie, K., and K. Hata. 2004. Turbulent energy balances inside short bubble formed on NACA 0012 airfoil. AIAA J. 42:1261-1264. 